International Journal of Biology, Pharmacy and Allied Sciences (IJBPAS)

'A Bridge Betueen Caboratory med QRendo'

Www.iibpas.com

EXTRACTION OF EMOTION CORRELATION OF PATIENTS BY USING MACHINE LEARNING MODELS

\title{
B.CHARITHA $^{1^{*}}$, M.V.P. CHANDRA SEKHARA RAO ${ }^{2}$ AND G.S.RAGHAVENDRA $^{3}$
}

Department of Computer Science and Engineering, RVR \& JC College of Engineering, Guntur, India

*Corresponding Author: B.Charitha; E Mail: charithabellam@gmail.com

Received $19^{\text {th }}$ July 2021; Revised $20^{\text {th }}$ Aug. 2021; Accepted $29^{\text {th }}$ Sept. 2021; Available online $1^{\text {st }}$ Nov. 2021

https://doi.org/10.31032/IJBPAS/2021/10.11.1009

\begin{abstract}
Emotion recognition has become a challenging task in the field of natural language processing since there is a large amount of data available on the Internet. Different methods are used to recognize emotion. In this paper, CNN-LSTM and logistic regression are used to recognize emotion. Most of the work in the field of artificial intelligence focuses only on the recognition of emotions, rather than exploring the reasons why emotions are not recognized or misrecognized. The correlation between emotions leads to the failure of emotion recognition. Here, we fill the gap between emotion recognition and emotion correlation. The emotion correlation is extracted by using confusion of emotion and evolution of emotion. Emotion correlation is based on the emotion recognition results of the machine learning models by using text.
\end{abstract}

Keywords: Affective computing, logistic regression, convolution neural network (CNN), long short-term memory (LSTM), deep neural networks, emotion correlation, emotion recognition, natural language processing (NLP)

\section{INTRODUCTION}

Emotions are a decisive aspect of the human condition. They penetrate into our social and professional lives, affect our thinking and behaviour, and profoundly shape our interpersonal relationships and social interactions. There are different levels of emotions: Individual emotion and public emotion. 
- Individual emotions are complex due to long-term individualized social experiences, interpersonal misunderstandings, and momentary external influences of emotions.

- Public emotion regarding social events is complicated for example: Information on social activities is posted online and covers various topics. Individualized netizens tend to focus on different aspects. Readers emotions can be diverse, giving different aspects of the same social event. If the reader is concerned about more than one aspect, your words may carry more than one emotion. The human emotions divided into five categories, that is, joy, sadness, anger, neutral, fear.

Nowadays, emotion recognition in the computer field is developing rapidly. Computers can uniformly recognize emotions and continue to learn new data quickly. Several studies using different approaches to detect human emotion aims to improve human-machine communication and make the interaction more efficient, usable and easy. In addition, the development of NLP and Computer-Brain Interface $(\mathrm{BCI})$ has become more and more interesting and challenging due to the massive growth of the Internet, especially social media [1]. Various machine learning models are used for emotion recognition.

Convolution Neural NetworkLong Short Term Memory (CNNLSTM) model: It combines the strengths of both architectures and proposes a new unified model for sentence representation and text categorization. It can capture both local characteristics of sentences as well as global and temporal semantics of sentences. We evaluate the proposed architecture on sentiment and question classification tasks. The test findings reveal that $\mathrm{CNN}$ LSTM outperforms both CNN and LSTM in these tasks and can produce great outcomes [2].

Logistic Regression model: It is a supervised machine learning model. The LR works on 5 emotional signals and the classifier is trained on a set of well-annotated emotional data. The LR algorithm works on training and testing a dataset of emotions. Before entering the dataset, several pre-processing steps are applied to the entered text. We used different Python libraries to implement the system [3].

Emotions are correlated rather than independent, which leads to the complexity of individual and public emotions. Emotion correlation mining will assist us in 
analyzing individual and public emotions for the following applications.

Social Media Communication: It is advantageous to generate low ambiguous messages that are empathetic to the information receiver for both news compilation and interpersonal communication. Emotion correlation mining can provide hints for the expression of desired emotions.

Public Sentiment Analysis: In social media, emotion variation plays a big role in netizens behavior understanding and anomalous event detection.

\section{Human Computer Interaction: Emotion contributes to improve $\mathrm{HCI}$, for example, social companion robots.}

Potential applications of emotion analysis have attracted much attention from researchers. However, most efforts are focused on emotion recognition, ignoring emotion correlation. In this paper we consider both emotion recognition and emotion correlation. The emotion recognition is performed by using machine learning models and the emotion correlation can be performed by using emotion confusion and emotion evolution.

Emotion confusion refers to the distance between emotions and it is performed by using absolute and relative confusion of emotion. The absolute confusion of an emotion represents average probabilities measured by the distance at which the emotion is confused with other emotions. The relative confusion of emotions is based on relative distance. Emotion evolution can be assessed using

$$
\begin{aligned}
& \text { i. One-step shift } \\
& \text { ii. Limited-step shifts and } \\
& \text { iii.Shortest path transfer. }
\end{aligned}
$$

\section{RELATED WORK}

In recent years, emotion analysis has attracted the attention of researchers. Khodijah Hulliyah in [1] mainly focuses on pattern analysis and classification techniques that can be used to process EEG signals to analyze emotion. Chunting Zhou in [2] have used C-LSTM model to evaluates the learned semantic sentence representations on sentiment classification and question type classification tasks with very satisfactory results. Fahad Mazed Alotaibi in [3] have used Logistic Regression, a classifier-based supervised learning approach is proposed to classify text into different emotional classes. Xinzhi Wang in [4] used linear regression and LMS (least mean squares) to solve the problem of social emotion detection of events according to microblogs, which is an important topic of public opinion mining on the background of big data. Saif $M$. Mohammad in [5] is about creating a new 
affect in tweets data set of more than 22,000 tweets. Ashwini Ann Varghese in [6] explains about strengths and weaknesses of different techniques adopted in the emotion recognition system was analyzed. Rohit Pathar in [7] have used deep convolution neural network to develop a smart vision for the device that can detect human face and recognize its emotion, in a single integrated module.

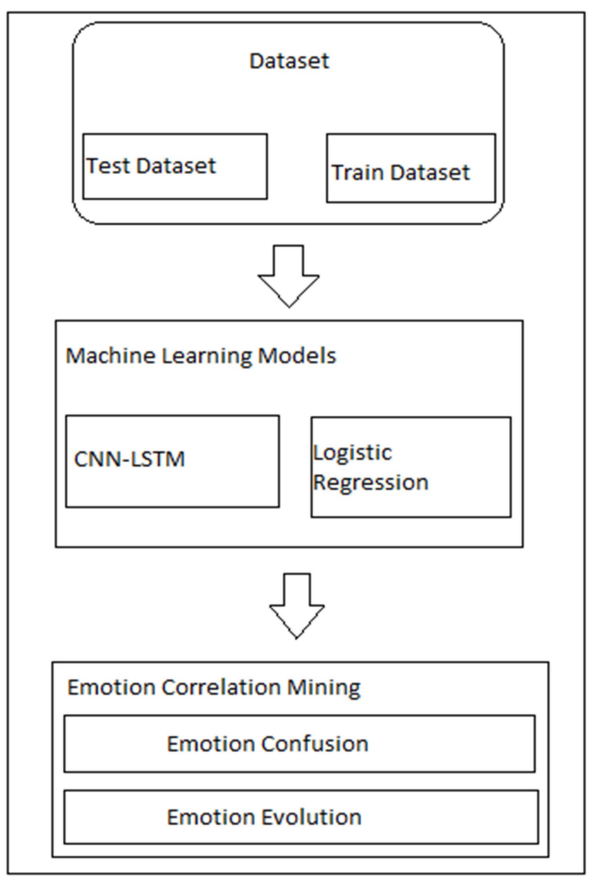

Figure 1: Extraction of emotion correlation

\section{Mounika Karna and Shadi Shaheen in $[8,9]$} have used various ontologies such as WordNet, Concept Net and different machine learning models like LSTM, SVM, Nested LSTM to recognize the emotion.Fan Xia in [10] have used deep learning models to summarize the techniques of emotion analysis and explicitly introduces the technology of emotion analysis.Dr. Sattar B in [11] explains about the effectiveness of a fuzzy logic controller and it can be improved by utilizing strategies in view of neural systems and genetic algorithms. Umar
Rashi and Forugh Mozafari in $[12,13]$ have used 3-turn conversation and different methods like VSM, keyword basis, STASIS to detect the emotion. Khodijah Hulliyah in [14] is about brain mapping and it is based on the polarity of the valence (V) and arousal (A) model into consideration to analyze the sentiment classification of text.R. Geetha Ramani in [15] explains about identifying the emotions from news articles into positive, negative, mixed or neutral through data preprocessing and data mining techniques. Wenkuan $\mathrm{Li}$ in [16] have used deep neural network models for 
text sentiment classification tasks by integrating sentiment linguistic knowledge into the neural network via a sentiment attention mechanism. Zafer Erenel in [17] explains about a new feature selection scheme and it is proposed to study the influence of selected features on emotion recognition from text. Shangsong Liang in [18] is about dealing issue with dynamic grouping of users in the context of short text streams.Liang Yao in [19] have used graph convolutional networks for text classification. Sara Azmin in [20] have used naive bayes classifier to detect emotion. K.Prasada Rao, M. V. P. Chandra Sekhara Rao, in [21] describes the framework that utilizes the Speech (Mel Frequency Cepstral Coefficients) features and Facial (Maximally Stable Extremal Regions) features to predict the emotions of a person through a systematic and scientific study. Seo-Hui Park in [22] have used a CNN model for emotion categorization using the emotion hashtag as an emotion label and then the embedding model that was built throughout the learning phase was extracted. Haji Binali in [23] have used a hybrid-based architecture and the SVM algorithm, which obtains a prediction accuracy of 95\%. Edward Chao-Chun Kao in [24] provides an overview of the burgeoning subject of emotion recognition from text, as well as a description of the current generation of detection algorithms and three main techniques are used to recognize emotion i.e. Keyword-based, learning-based, and hybrid based. Varun Sundaram in [25] have used a new approach for emotion detection based on TFIDF and it is metric that reflects the value of a word in a document. Zhi Teng in [26] provides a new scheme to detect emotion from text through classification with the Rough set theory and support vector machine.

\section{MODEL FRAMEWORK}

The purpose of this article is to determine the potential and meaningful correlations between sentiments from online messages. The Emotions classification models in Section III-A and III-B focus on distinguishing emotion orientation from input text. The calculation part for emotion correlation is described in section III-C and III-D, and the overall framework is shown in Figure 1.

A. CNN-LSTM: CNN-LSTM model is employed for emotion recognition and it is a combination of CNN and LSTM. We create a basic end-to-end unified architecture by feeding the output of a single-layer $\mathrm{CNN}$ into the LSTM to take advantage of the benefits of CNNs and RNNs. CNN is built on top of pre-trained word vectors from huge untagged text data to learn higher level representations of n-grams. Then, to learn sequential correlations from toplevel sequence representations, CNN's 
feature map was organized into sequential window features to serve as input to the LSTM. This way, instead of building LSTM directly from the input sentence, we first transform each sentence into sequential window features to aid in the disentanglement of factors of variation within sentences. Before feeding the neural network, we use sequence based input rather than depending on syntactic parse trees, so our model does not rely on any external language knowledge or expensive preprocessing. In this model, the length of an input text can be either short or long. The output of the models is one of the five kinds of emotions, that is, love, joy, anger, sadness, fear, and surprise. The calculation process can be divided into two parts(part 1, and part 2).

Part 1: It mainly focuses on feature processing which transforms the original features into dense vector information. There are four operations: vector lookup; window sliding; convolutional calculation; and rectified linear unit activation.

Part 2:It mainly focuses on emotion calculation. There are five operations, that is, LSTM calculation, dropout operation, average calculation, fully connected layer, and softmax. Recurrent neural networks (RNNs) are powerful for sequence processing tasks, such as text classification.

\section{B. Logistic Regression:}

The Logistic Regression (LR) classifier is used to identify emotions from input text, and it also uses a supervised machine learning approach to perform emotion classification.

Text-based emotions detected and classified by using logistic regression (LR) and it is achieved by performing training and testing.

\subsection{1: Training and testing the dataset}

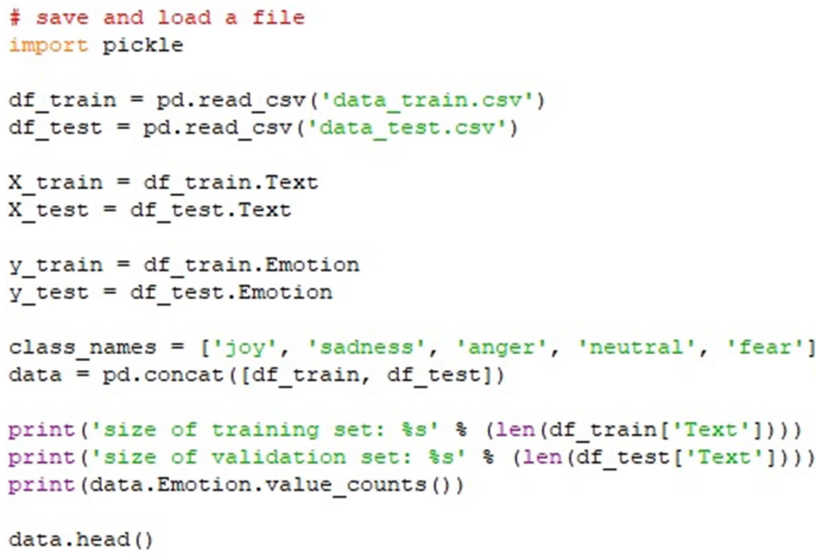

Figure 2: Training and testing the dataset 


\subsection{2: Implementation for logical regression}

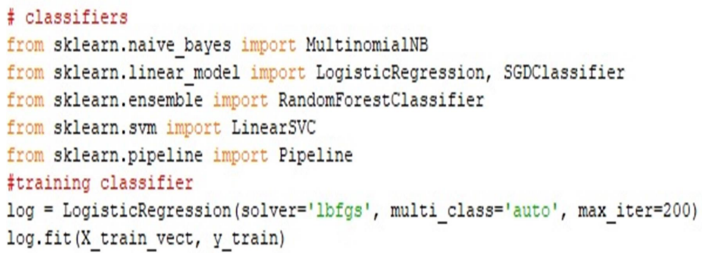

\section{Confusion of Emotion:}

Figure 3: Implementing code for logistic regression

Let $e_{g}$ represents the feature of emotion $c_{g}$. Confusion degree refers to the distance between emotions and it is obtained by subtracting the corresponding columns. The Closer distances represent a greater degree of confusion. For example, subtract $e_{0}, \ldots, e_{m_{0}-\mathbf{1}}$ from $e_{g}$. Then the emotion $c_{g}$ centered distance matrix is obtained and represented by

$$
X_{3}^{g}=\left[d_{0}^{g}, d_{1}^{g}, \ldots, d_{m_{0}-1}^{g}\right] \epsilon R^{\left(m_{1}+1\right) m_{0}}
$$

Here, $d_{i}^{g} \in X_{3}^{g}$ represents a column vector. $d_{i}^{g}$ represents distance among emotion $c_{i}$ and emotion $c_{g}$, from $m_{1}+1$ points of view.

1) Absolute Confusion Degree: The absolute degree of confusion represents the possibility that one emotion is confused with another. An increase in the degree of confusion of $c_{g}$ emotion with any other emotion will increase the absolute confusion degree $c_{g}$

$$
A^{g}=\frac{\sum_{i=1}^{m_{0}} d_{i}^{g}}{\left(m_{1}+1\right) m_{0}}
$$

Here $A^{g}$ represents a farther distance among $c_{g}$ and all other emotions.

2) Relative Confusion Degree: For a matrix $X_{3}^{g}$, the five elements in each row are sorted in ascending order, i.e. $X_{4}^{g}=$ $\operatorname{ARGSORT}\left(X_{3}^{g}\right)$. The sequence matrix $X_{4}^{g}$ is given by $m_{0}-1$

$$
X_{4}^{g}=\left[c e_{0}^{g}, c e_{1}^{g}, \ldots, c e_{m_{0}-1}^{g}\right]
$$

$\epsilon R^{\left(m_{1}+1\right) m_{0}}$

Maximum confusion with the emotion $c_{g}$, the first column elements. In contrast, the last column element $\left(c e_{m_{0}-1}^{g}\right)$, which represents the emotion, has minimal confusion with the emotion $c_{g}$, the first column elements.

For a matrix of sequence $X_{4}^{g}$, if all the elements of a column are identical, this means that the perspectives $m_{1}+1$ are the same. A reliable result is obtained. That is, the more similar elements there are in a column, the more reliable the result. The introduction of information entropy eliminates unreliable results.

$$
H(U)=E\left[-\log p_{i}\right]=-\sum_{i=1}^{n} p_{i} \log p_{i}
$$

Here, $p_{i}$ represents the proportion of a emotion in a column. Entropy indicates the degree of chaos. Lower information entropy refers to higher consistency, i.e. 
a more reliable result. Therefore, columns of $X_{4}^{g}$, which have lower than average information entropy, are extracted and analyzed.

D. Emotion Evolution: The evolution of emotion is directional and it mainly focus on emotion shift during social events propagation. Emotion evolution can be evaluated from the following aspects.

Here, $X_{1}^{(k)}$ is the emotion correlation matrix. $\operatorname{Prob}_{n}\left(c_{i}, c_{j}\right)$ represent the probability that $c_{i}$ is recognized as $c_{j}$ after $\mathrm{n}$ steps. Trace $_{n}\left(c_{i}, c_{j}\right)$ represent the corresponding path. Every shift step corresponds to a misjudgment between two emotions. $\operatorname{Prob}_{n}\left(c_{i}, c_{j}\right)$ is the multiplication of misjudgment probability of every shift step.

1) One-step shift: The misunderstanding emotion pair is figured out given an emotion $c_{i}$. According to the matrix $X_{1}^{(k)}$, compare $\operatorname{Prob}_{1}\left(c_{i}, c_{j}\right)$ in terms of each emotion $c_{j}$. Then, the top pair is the two emotions with the maximal $\operatorname{Prob}_{1}\left(c_{i}, c_{j}\right)$. The trace is denoted as $\operatorname{Trace}_{1}\left(c_{i}, c_{j}\right)=c_{i} \rightarrow c_{j}$

$\max \left\{\operatorname{Prob}_{1}\left(c_{i}, c_{j}\right) \mid \mathbf{j}=0, \ldots\right.$

$\left., m_{0}-1, \mathbf{j} \neq 1\right\}$.
2) Limited-step shift: Under the three situations of given initial emotion, final emotion, initial and final emotion, observe the evolution of the emotion in limited steps.

To extract the most possible shift path, the probability product $\operatorname{Prob}_{n}\left(c_{i}, c_{j}\right)$ is calculated and compared. However, after $n$ shift steps, the product maybe close to zero. As a result, the logarithm of the matrix $X_{1}^{(k)}$ is used.In particular, the logarithm of the misjudgment probability for each shift is synthesized and used, which is positively correlated with $\operatorname{Prob}_{n}\left(c_{i}, c_{j}\right)$.

Let the initial and final emotions be called $c_{\text {ini }}$ and $c_{\text {fin }}$ respectively. When $c_{i n i}$ or $c_{f i n}$ is given, there are $n^{m_{0}-1}$ potential shift line types in $\mathrm{n}$ displacement steps with the existence of a judgment error. When given both $c_{\text {ini }}$ or $c_{f i n}$, there are $n^{m_{0}-2}$ types of potential compensating paths. In the above three conditions, the moving line with the greatest probability is drawn. In other words, at each stage of change, the emotion that is most likely to be misjudged with the previous emotion will be selected for the emotional stream. The above process is shown as follows:

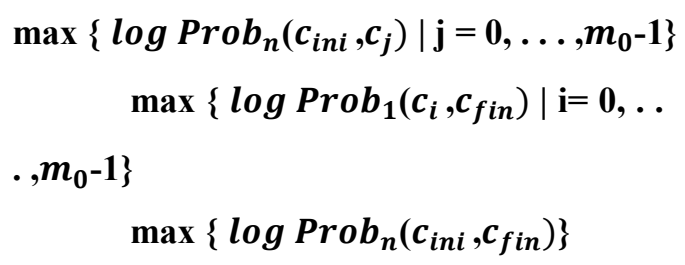


3) Shortest path transfer: Given the initial and final emotion, the shortest path between two emotions is observed. The shortest path from emotion $c_{i n i}$ to $c_{\text {fin }}$ is either one or $\mathrm{n}$ steps through other emotions. Specifically, the trace with the maximal probability of misadjustments among one to $\mathrm{n}$ shift steps is the shortest one

$\max \left\{\max \left\{\log \operatorname{Prob}_{n}\left(c_{\text {ini }}, c_{\text {fin }}\right)\right\} \mid \mathrm{n} \in \mathrm{N}^{*}\right\}$

\section{EXPERIMENTS}

The dataset is collected from one of the most popular social network platforms, news channel and then the dataset is spilt into train dataset and test dataset. Here, two machine learning models, $\mathrm{CNN}$ LSTM and logistic regression are used for emotion recognition. In both models, given the same input text, the result of these models may be same or different because the process for these models is different but the output must be any one of the five kinds of emotions, that is joy, sadness, anger, neutral, fear. For example, in Figure 4 and Figure 5 the input is given for CNN-LSTM and logistic regression is same and the output for these models is same (output must be any one of the five kinds of emotion). Similarly, in Figure 6 and Figure 7 the input is given for CNN-LSTM and logical regression is same but the output for these models is different. To avoid these problems, we use emotion correlation.

$\left[\begin{array}{lllll}34 & 23 & 38 & 505 & 38\end{array}\right]$

$\left[\begin{array}{lllll}72 & 43 & 53 & 42 & 466\end{array}\right]$

enter text I was very happy when my scholarship to continue studying at university was approved after it had been cancelled.

I was very happy when my scholarship to continue studying at university was approved after it had been cancelled. ['joy'] $\gg$

Figure 4: Output for Logistic Regression

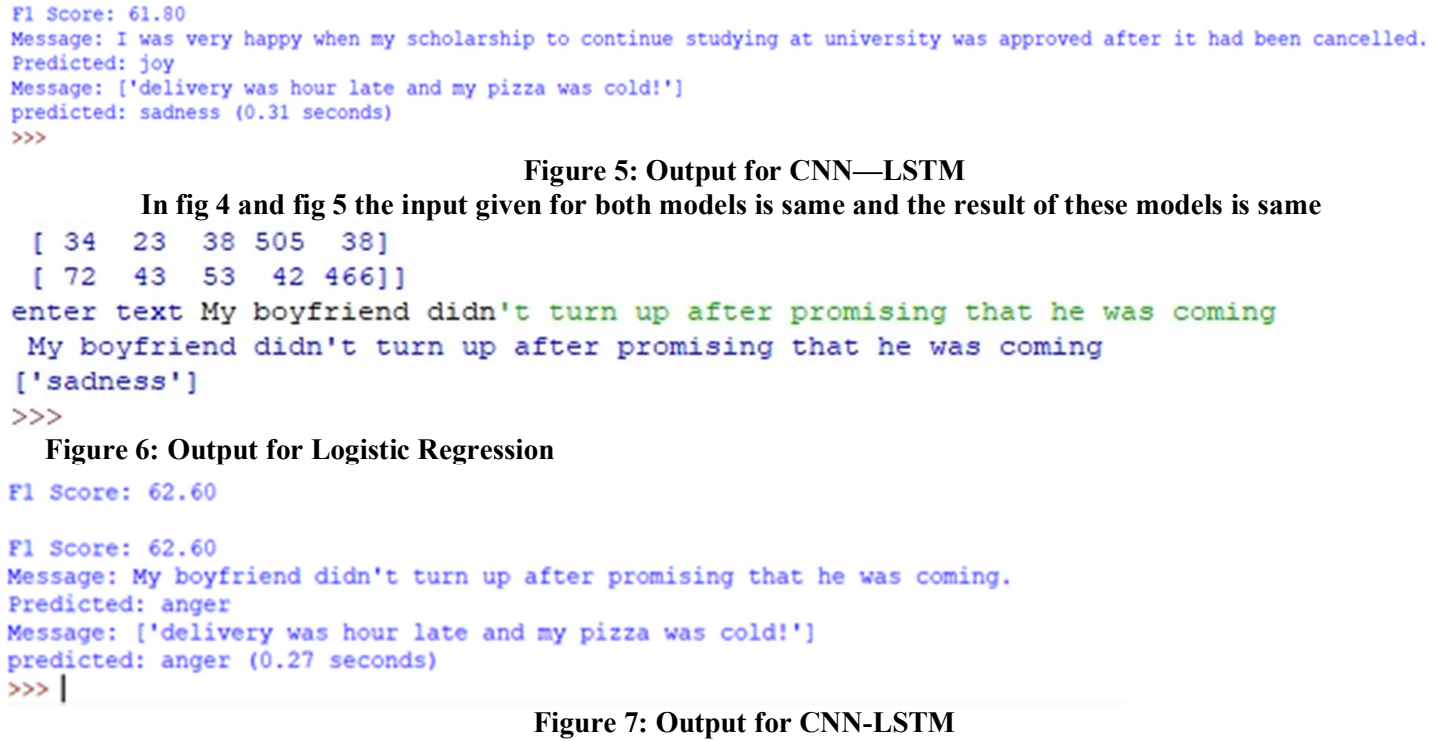

Figure 5: Output for CNN-LSTM

In fig 4 and fig 5 the input given for both models is same and the result of these models is same

Figure 7: Output for CNN-LSTM

In Figure 6 and Figure 7 the input for both models is same but the result of these models are different 
Emotion correlation mining is defined as emotion confusion and emotion evolution.

Emotion confusion refers to the distance between emotions due to mutual misjudgment between two emotions. In emotion confusion we use normalized confusion matrix to show the performance of a classifier or model that shown below.

Figure 8: Showing the program output showing the normalized confusion matrix of Logistic Regression
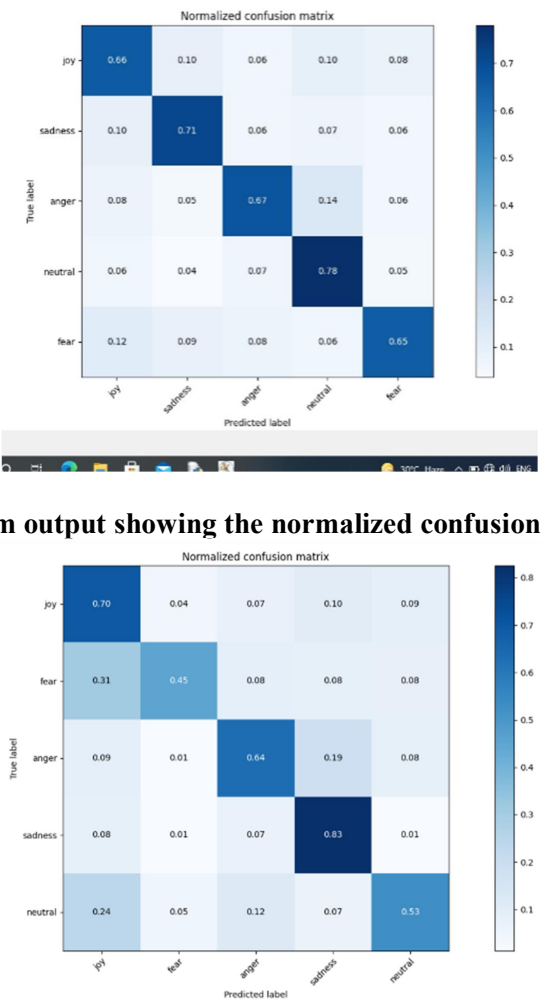

$$
\text { "C }
$$

Figure 9: Screenshot the program output showing the normalized confusion matrix of CNN-LSTM

In Figure 8 and Figure 9, a row represents an instance of the actual class whereas a column represents an instance of the predicted class and the diagonal element values represent the degree of correctly predicted classes. The confusion is expressed by the false classified off diagonal elements, which are mistakenly confused with other emotions.

The Emotion confusion is performed by using confusion degree. The confusion degree refers to distance among emotions. There are two confusion degrees, such as absolute confusion degree and relative confusion degree. The calculation part for emotion confusion is defined in III - C.

- Absolute confusion degree represents the average probability measured by the distance that the emotion is confused with all other emotions. For example, the probability that love is mistaken as other emotions and other emotions are misjudged as love. Increasing any of the factors will increase the absolute confusion of 
emotion love.

- Relative confusion degree is based on the relative distance. For example, if the gap between fear and surprise is shorter than the gap between fear and anger, this shows that in terms of fear, the relative confusion degree of surprise is higher than that of anger. Fear and surprise are more likely to be confused. Basically, there is only one absolute confusion value for a given emotion, but many relative confusion values for that certain emotion, with each other.

Evolution of emotion helps us to avoid misjudgment of emotion, confusion of emotion and it also helps us to find shortest path between two emotions. The calculation part for emotion evolution is described in III -D.

\section{CONCLUSION}

In this paper, the data are collected from various sources like social media and newspaper. The emotion recognition is done by using CNN-LSTM and Logistic Regression models. In both models, the result of these models may be same or different for the given same input text. In order to avoid misjudgment of emotion and confusion of emotion, emotion correlation is used. It is observed that accuracy of the proposed method using CNN-LSTM model is $62 \%$ where as Logistic Regression the accuracy of model is increased by $25 \%$.

\section{REFERENCES}

[1] Khodijah Hulliyah, Normi Sham Bt. Awang Abu Bakar, "Analysis of Emotion Recognition Model Using Electroencephalogram (EEG) Signals Based on Stimuli Text," Turkish Journal of Computer and Mathematics Education, Vol.12, No.3, PP-13841393, 27th Jan 2021.

[2] Chunting Zhou, Chonglin Sun, Zhiyuan Liu, Francis C.M. Lau1,"A C-LSTM Neural Network for Text Classification," 30th Nov 2015 .

[3] Fahad MazaedAlotaibi, "Classifying text-based emotions using logistic regression," VFAST Transactions on Computer Sciences, Vol. 7, No. 1, JanuaryDecember, 2019.

[4] Xinzhi Wang, Xiangfeng Luo, Jinjun Chen, "Social sentiment detection of event via microblog" 2013 IEEE 16th International Conference on Computational Science and Engineering.

[5] Saif M. Mohammad, Saif M. Mohammad, Mohammad Salameh, Svetlana Kiritchenko, "Affect in Tweets", The 12th International 
Workshop on Semantic Evaluation (SemEval-2018).

[6] Ashwini Ann Varghese, Jacob P Cherian, Dr. Jubilant J Kizhakkethottam, "Overview on Emotion Recognition System", 2015 International Conference on Soft-Computing and Network Security (ICSNS -2015), Feb. 25 27, 2015, Coimbatore, INDIA.

[7] RohitPathar, Abhishek Adivarekar, Arti Mishra, AnushreeDeshmukh, "Human Emotion Recognition using Convolutional Neural Network in Real Time", IEEE, 2019.

[8] Mounika Karna, D.Sujitha Juliet, R.Catherine Joy, "Deep learning based Text Emotion Recognition for Chatbot applications", the Fourth International Conference on Trends in Electronics and Informatics (ICOEI 2020)IEEE Xplore Part Number: CFP20J32ART; ISBN: 978-1-7281-5518-0.

[9] ShadiShaheen, Wassim ElHajj,Hazem Hajj, Shady Elbassuoni, "Emotion Recognition from Text Based on Automatically Generated Rules", 2014 IEEE International Conference on Data Mining Workshop.

[10] Fan Xia, Zhi Zhang, "Study of Text Emotion Analysis Based on
Deep Learning", The National Natural Science Foundation of China, IEEE, 2018.

[11] Dr. Sattar B. Sadkhan, Ahmed Dheyaa Radhi, "Fuzzy Logic used in Textual Emotion Detection", International Science Conference on Multidisciplinary in IT and Communication Science and Technologies, IEEE, 2017.

[12] Umar Rashi, Muhammad Waseem Iqbal, Muhammad AkmalSkiandar, Muhammad QasimRaiz, Muhammad Raza Naqvi, Syed KhuramShahzad, "Emotion Detection of Contextual Text using Deep learning", Auckland University of Technology, December 21,2020 from IEEE Xplore.

[13] Forugh Mozafari, Hooman Tahayori,"Emotion Detection by Using Similarity Techniques", 2019 7th Iranian Joint Congress on Fuzzy and Intelligent Systems (CFIS).

[14] Khodijah Hulliyah, Normi Sham Awang Abu Bakar, Amelia Ritahani Ismail, "Emotion Recognition and Brain Mapping for Sentiment Analysis", IEEE, 2017. 
[15] R. Geetha Ramani, M. Naveen

Kumar,

Lakshmi

Balasubramanian, "Identification

of Emotions in Text Articles

through Data Pre-Processing and

Data Mining Techniques".2016

International Conference on

Advanced Communication

Control and Computing

Technologies (ICACCCT).

[16] Wenkuan Li, Peiyu Liu, Qiuyue Zhang and Wenfeng Liu, "An Improved Approach for Text Sentiment Classification Based on a Deep Neural Network via aSentiment Attention Mechanism", Future Internet,2019.

[17] Zafer Erenel, Oluwatayomi Rereloluwa Adegboye, Huseyin Kusetogullari, "A New Feature Selection Scheme for Emotion Recognition from Text", Applied Sciences, 2020.

[18] Shangsong Liang, Zhaochun Ren, Emine Yilmaz, Evangelos Kanoulas, "Collaborative User Clustering for Short Text Streams", The Thirty-First AAAI Conference on Artificial Intelligence (AAAI-17), 2017.

[19] Liang Yao, Chengsheng Mao, Yuan Luo, "Graph Convolutional Networks for Text
Classification", The Thirty-Third AAAI Conference on Artificial Intelligence (AAAI-19), 2019.

[20] Sara Azmin, Kingshuk Dhar, "Emotion Detection from Bangla Text Corpus Using Naïve Bayes Classifier", 4th International Conference on Electrical Information and Communication Technology (EICT), 20-22 December 2019, Khulna, Bangladesh.

[21] Seo-Hui Park, Byung-Chull Bae, Yun-Gyung Cheong, "Emotion Recognition from Text Stories Using an Emotion Embedding model", International Conference on Big Data and Smart Computing, IEEE, 2020.

[22] Haji Binali, Chen $\mathrm{Wu}$, Vidyasagar Potdar, "Computational Approaches for Emotion Detection in Text", 4th International Conference on Digital Ecosystems and Technologies , IEEE, 2010.

[23] Edward Chao-Chun Kao, TingHao Yang, Chang-Tai Hsieh, Von-Wun Soo, "Towards Textbased Emotion Detection", International Conference on Information Management and Engineering, 2009. 
[24] Varun Sundaram, Saad Ahmed, Shaik Abdul Muqtadeer, R.Ravinder Reddy, "Emotion Analysis in Text using TF-IDF", $11^{\text {th }}$ I international conference on cloud computing, IEEE, 2021.
[25] Zhi Teng, Fuji Ren, Shingo Kuroiwa, "Emotion Recognition from Text based on the Rough Set Theory and the Support Vector Machines”, IEEE, 2007. 